Konstanzer Online-Publikations-System (KOPS)

URL: http://www.ub.uni-konstanz.de/kops/volltexte/2008/4796/

URN: http://nbn-resolving.de/urn:nbn:de:bsz:352-opus-47969

\author{
Aus dem Institut für Pfanzenpathologie und Pflanzenscbutz \\ der Universität Göttingen
}

\title{
Der Infektionsverlauf von Uromyces pbaseoli bei anfälligen und resistenten Bohnensorten
}

\author{
Von \\ K. Mendgen
}

Mit 21 Abbildungen

Eingegangen am 4. November 1977

Der Infektionsverlauf bei Rostpilzen ist allgemein bekannt. Zahlreiche Untersuchungen (siehe MENDGEN 1973) deuten darauf hin, daß jeder Rostpilz auf die für ihn typische Art und Weise in das Blatt eindringt und dort Haustorien ausbildet.

Bei Uromyces phaseoli var. vignae, dem Rost der Kuhbohne, wurde genau beschrieben, wie sich der Keimschlauch der Uredospore auf der Blattoberfläche verhält, die Spaltöffnung aufsucht und durch diese ins Blattinnere eindringt (HEATH 1974). Auf Grund der unterschiedlichen Oberflächenstruktur der Epidermis „findet" der Keimschlauch die Spaltöffnung von Nichtwirtspflanzen weniger häufig: Bei anfälligen und resistenten Wirtspflanzen liegen aber offensichtlich keine Interaktionen vor, die zu verringertem Wachstum auf der Blattoberfläche oder zu verringertem Erfolg bei der Penetration durch die Spaltöffnung führen (HEATH 1974). Erst wenn der Pilz in das Blattinnere eingedrungen ist, kommt es während des Infektionsverlaufs zu Unverträglichkeitsreaktionen, die zum Absterben des Pilzes oder - und - der Wirtszellen führen (HEATH und HeATH 1971, HeATH 1972, 1974).

Es ist zu vermuten, daß bei dem nahe verwandten Bohnenrost (Uromyces phaseoli var. typica) die wirtsspezifischen Interaktionen, die zur Verträglichkeit oder Unverträglichkeit führen, ebenfalls während der ersten Stunden des Pilzwachstum im Blattgewebe stattfinden (MARTE und MonTalbin 1972).

Es liegen jedoch noch keine Untersuchungen darüber vor, wie vielfältig die Wirtsreaktionen bei unverträglicher Kombination sein können und in welcher Variationsbreite sie sich ereignen. Dies erscheint besonders wichtig, weil die neuesten Untersuchungen über Infektionsvorgänge bei Rostpilzen und beim 
echten Mehltau größtenteils mit isogenen Linien durchgeführt wurden (LITTLEpield 1973, Skipp und Samborski 1974, Ellingboe 1972). Dadurch ist zwar bekannt, wie die einzelnen bekannten Resistenzgene wirken könnten, die Möglichkeiten der vielen anderen vorhandenen Resistenzgene wird aber nicht erfaßt.

Für solche Untersuchungen erscheint die Kombination Bohne.(Phaseolus vulgaris) und Bohnenrost (Uromyces phaseoli var. typica) besonders geeignet, weil wir auf Grund der vorhandenen unterschiedlichen Resistenzgene (ZAUmeyer und Meiners 1975, Ogle und Johnson 1974) die Möglichkeit erhalten, die Variationsbreite der unverträglichen Kombinationen von Bohne und Bohnenrost zu erfassen.

\section{Material und Methoden}

\section{Anzucht der Pflanzen, Inokulation und Prüfung auf Anfälligkeit}

Die Bohnen wurden im Lichtthermostaten bei $21^{\circ} \mathrm{C}$ und etwa $6000 \mathrm{Lux} 16 \mathrm{~h} / \mathrm{Tag}$ angezogen. Zehn Tage nach der Aussaat wurden die Primärblätter mit einer Sporensuspension von Uromyces phaseoli (Pers.) Wint. var. typica Arth. (erhalten vom Institut voor Plantenziektenkundige Onderzoek, Wageningen) besprüht und für $24 \mathrm{~h}$ in einer Feuchtkammer gehalten. Die Sporen in der Sporensuspension waren während $1 \mathrm{~h}$ in fließendem Wasser gespült und ihre Konzentration so eingestellt worden, daß bei der anfälligen Sorte Favorit etwa vier Pusteln $/ \mathrm{cm}^{2}$ Blattfläche entstanden. Die Reaktionen dér Bohnenblätter und die Entwidklung des Rostes wurden täglich beobachtet.

\section{Herkunft des Saatgutes ${ }^{1}$ )}

Die Bohnensorten (oder Linien) Favorit, Warox, 07054, 046, 08, 057, 017, 07036, 010, 5961 erhielten wir von Herrn Lörke und Herrn Dr. Buddenschön, Firma Van Waveren, Rosdorf über Göttingen;

die Bohnensorte Tui erhielten wir von Herrn Dr. MEINERs, USDA, Beltsville, USA;

die Bohnensorten Golden Gate Wax, 814, Greenleaf erhielten wir von Herrn Dr. RuDOLPH und Herrn Dr. WOLF, Universität Göttingen;

die Bohnensorten Epicur, Redlands Greenleaf A, Redlands Greenleaf B, Redlands Greenleaf C erhielten wir von Dr. G. Purss, Dept. of Primary Industries, Indooroopilly, Brisbane, Australien;

die Bohnensorte NB 518 erhielten wir von Herrn Dr. D. M. Murunya, University of Nairobi, Kenya;

die Bohnensorten Chiapas 36-2 und CIAT 602281 PI 311-930 von Herrn Dr. G. E. Gatvez E. CIAT, Cali, Kolumbien.

\section{Aufarbeitung für die Lichtmikroskopie}

Fïr die lichtmikroskopische Untersuchung des Infektionsvorganges wurde eine Sporensuspension $2 \mathrm{~h}$ in Leitungswasser gewaschen. Dann wurden die gewaschenen Sporen mit einem Pinsel in gleidımäßiger Schicht auf die Blattoberfläche aufgetragen. Dadurch wurde eine sehr dichte Infektion des Blattes erreicht. 12,13,14,16, 21, 28 und 40 Stunden nach Infektion (h.p.i.) wurden Blattstücke $(0,5 \times 2 \mathrm{~mm})$ ausgeschnitten und $60 \mathrm{~min}$ bei $20^{\circ} \mathrm{C}$ in einer Lösung von $3,5 \%$ Glutaraldehyd, $2 \%$ Saccharose und $0,08 \mathrm{M}$ Cacodylatpuffer $\mathrm{pH} 7,1$ vorfixiert. Danach wurden die Proben in Puffer gewaschen, mit $2 \%$ Osmiumsäure nachfixiert, über eine Alkoholreihe entwässert und in Epoxidharz (SPUR R 1969) eingebettet. Von den Proben wurden $2 \mu \mathrm{m}$ didke Querschnitte mit Glasmessern angefertigt, auf Wasser geglättet, in W15 (Zeiss, Oberkochen) eingebettet und die Deckgläser mit Uhu-Plus umrandet.

1) Den obengenannten Herren danke ich für die freundliche Uberlassung des Saargutes. 


\section{Lichtmikroskopische Auswertung}

Von jeder Probe wurden mindestens 200 (bis 500) Schnitte in Serie angefertigt. In den einzelnen Schnittserien wurden 10 bis 20 Infektionsvorgänge des Pilzes verfolgt und diese gezeichnet. Die Zeichnungen wurden begutachtet und daraufhin ein Schema der Infektion für jede Probe entworfen. Diese schematischen Zeichnungen sind in den Abbildungen wieder-
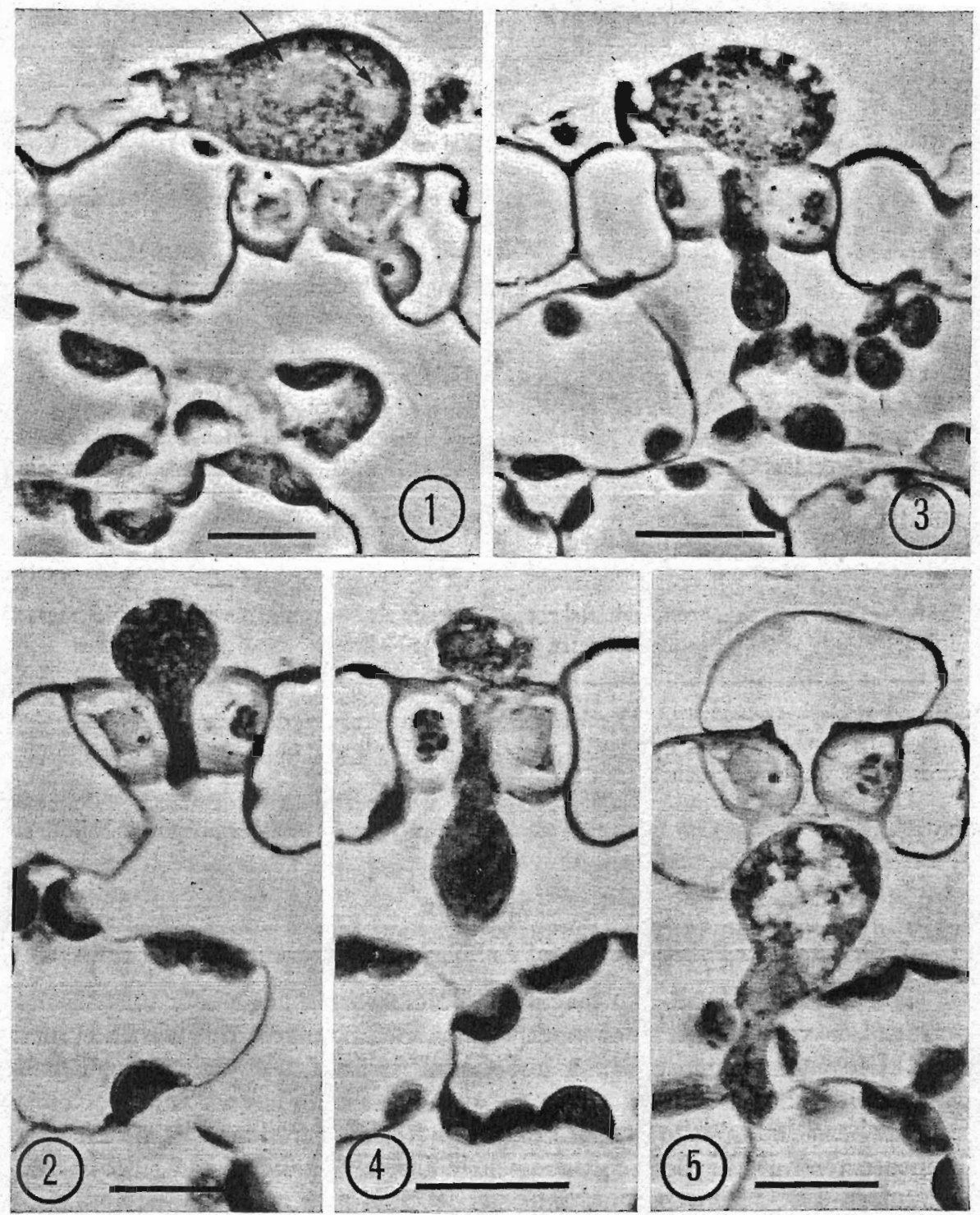

Abb. 1 bis 5. Verschiedene Stadien des Infektionsvorganges, die im Zeitraum von 12 bis 16 h.p.i. angetroffen wurden. - Abb.1. Appressorium über den Schließzellen (Pfeile deuten auf die Zellkerne. - Abb 2. Appressorium mit Infektionskeil, quer. - Abb. 3. Appressorium mit Infektionskeil, das substomatäre Vesikel ausbildend, längs. - Abb.4. Appressorium mit Infektionskeil und substomatärem Vesikel, quer. - Abb. 5. Leeres Appressorium und leerer Infektionskeil, durch ein Septum vom fertigen substomatären Vesikel abgeteilt 
Tabelle

Symptome auf den Primärblättern der

\begin{tabular}{|c|c|c|c|}
\hline Sorte & 3. d.p.i. & 4. d.p.i. & 5. d.p.i. \\
\hline Favorit & leichte Chlorosen & Chlorosen & Chlorosen \\
\hline Golden Gate Wax & leidite Chlorosen & Chlorosen & Chlorosen \\
\hline 07054 & - & - & - \\
\hline 046 & & leichte Chlorosen & \\
\hline 814 & & Chlorosen & \\
\hline Greenleaf & wenig Nekrosen & vermehrt Nekrosen & \\
\hline 08 & - & - & - \\
\hline 057 & - & vereinzelt Chlorosen & vereinzelt Chlorosen \\
\hline Chiapas 36-2 & - & - & - \\
\hline Warox & - & - & Nekrosen \\
\hline 017 & Nekrosen & größere Nekrosen & Nekrosen \\
\hline Tui & - & - & Nekrosen \\
\hline Ciat 602281 & & Nekrosen & Nekrosen \\
\hline Redlands Greenleaf A & kleine Nekrosen & kleine Nekrosen & kleine Nekrosen \\
\hline Redlands Greenleaf B & - & - & Chlorosen u. Nekrosen \\
\hline Redlands Greenleaf C & - & 一 & $\begin{array}{l}\text { Chlorosen und wenig } \\
\text { Nekrosen }\end{array}$ \\
\hline NB 518 & - & - & - \\
\hline 07036 & - & - & - \\
\hline 010 & - & - & - \\
\hline 5961 & vereinzelt Nekrosen & vereinzelt Nekrosen & vereinzelt Nekrosen \\
\hline Epicur & große Nekrosen & große Nekrosen & große Nekrosen \\
\hline
\end{tabular}

gegeben. Wenn unterschiedliche Reaktionen auftraten, wurden diese in den kreisförmigen Einsätzen der Abbildungen dargestellt und das Verhältnis der unterschiedlichen Reaktionen zueinander in Zahlen angegeben.

Die photographischen Aufnahmen erfolgten mit einer planapochromatischen Olimmersion 100 (Leitz, Wetzlar) im Phasenkontrast. Die Länge des Maßstabes auf den Abbildungen entspricht $10 \mu \mathrm{m}$.

\section{Ergebnisse}

\section{Prüfung der Bohnensorten auf Anfälligkeit gegenüber dem Bohnenrost}

Die Anfälligkeit aller angegebenen Bohnensorten gegenüber unserer Bohnenrostrasse wurde unter den angegebenen Bedingungen vergleichend untersucht. Die Symptomausprägung auf den Primärblättern zeigt die Tabelle. Danach werden die Sorte Favorit als anfällig, die anderen Bohnensorten als resistent bezeichnet (siehe STRAIB 1943). Die Reaktionen der meisten resistenten Bohnensorten auf die Bohnenrostrassen in ihren Anbaugebieten sind bekannt (Mukunya 1975, Meiners und Rogers 1975, Burga et al. 1975, Ogle und JOHNSON 1974).

\section{Der Infektionsverlauf bei der anfälligen Sorte Favorit}

Um genügend Infektionsvorgänge in den Schnittserien zu finden, wurden die Sporen für die lichtmikroskopischen Untersuchungen in einer gleichmäßigen 


\begin{tabular}{|c|c|c|}
\hline 6.+7.d.p.i. & 8. d.p.i. & 9. d.p.i. \\
\hline erste Pusteln & Pusteln & Pusteln \\
\hline Chlorosen & Chlorosen & Chlorosen und Pusteln \\
\hline - & - & keine Reaktion \\
\hline leichte Chlorosen & & leichte Chlorosen \\
\hline Chlorosen & Chlorosen & Chlorosen \\
\hline Nekrosen und Chlorosen & & vereinzelt Pusteln \\
\hline 一 & & leichte Chlorosen \\
\hline vereinzelt Chlorosen & vereinzelt Chlorosen & $\begin{array}{l}\text { vereinzelt Chlorosen und } \\
\text { Nekrosen }\end{array}$ \\
\hline Chlorosen & Chlorosen & Chlorosen \\
\hline Nekrosen & Nekurosen & Nekrosen \\
\hline Nekrosen & Nekrosen & Nekrosen \\
\hline Nekrosen & Nekrosen & Nekrrosen \\
\hline Nekrosen & Nekrosen und Pusteln & Nekrosen und Pusteln \\
\hline Nekrosen & Nekrosen und Chlorosen & Nekrosen und Chloroseh \\
\hline Chlorosen und Nekrosen & Chlorosen und Nekrosen & Chlorosen und Nekrosen. \\
\hline Chlorosen und wenig Nekrosen & Chlorosen und wenig Nekrosen & Chlorosen u. wenig Nekrosen \\
\hline - & - & keine Reaktion \\
\hline- & - & keine Reaktion \\
\hline - & - & leichte Chlorosen \\
\hline vereinzelt Nekrosen & vereinzelt Nekrosen & vereinzelt Nekrosen \\
\hline große Nekrosen und Chlorosen & Nekrosen & Nekrosen, Blatt stirbt ab \\
\hline
\end{tabular}

Schicht auf die Blattunterseite aufgetragen. Dadurch entstand ein dichtes Myzel aus Keimschläuchen und es sind keine Aussagen über das Verhalten des Pilzes auf der Epidermis möglich. Erst wenn der Keimschlauch das Appressorium ausdifferenziert, wird ein Infektionsvorgang weiter verfolgbar. Diese Technik ermöglicht jedoch, das Eindringen des Pilzes in die Atemhöhle an Schnittserien zu verfolgen:

Das Appressorium wird vom Keimschlauch etwa 12 Stunden nach Inokulation ausdifferenziert. Es ist eiförmig (Abb. 1) und verhakt sich mit seiner Unterseite in den Vorhofleisten der Schließzellen (MENDGEN 1973). Stündliche Probenahmen zeigten in einem Zeitraum von 12 bis 16 h.p.i. die folgenden Stadien: Appressorium (Abb. 1), Appressorium mit Infektionskeil (längs Abb. 2, quer Abb. 3), halbleeres Appressorium mit Infektionskeil und kleinem substomatärem Vesikel (Abb. 4), und leeres Appressorium mit substomatärem Vesikel und entstehender Infektionshyphe (Abb. 5).

Das Appressorium hat zwei bis vier Kerne (siehe auch STAPLES et al. 1975), die jedoch mit unserer Technik nur schwer im dichten Plasma erkennbar waren (Abb. 1). Die Ausbildung des Infektionskeils wird durch kleine Bläschen, die unter der Oberseite des Appressoriums entstehen, eingeleitet (Abb. 2). Wenn das Plasma durch den Infektionskeil in das entstehende substomatäre Vesikel fließt, vereinigen sich diese Bläschen zu einer Vakuole im Appressorium, die 

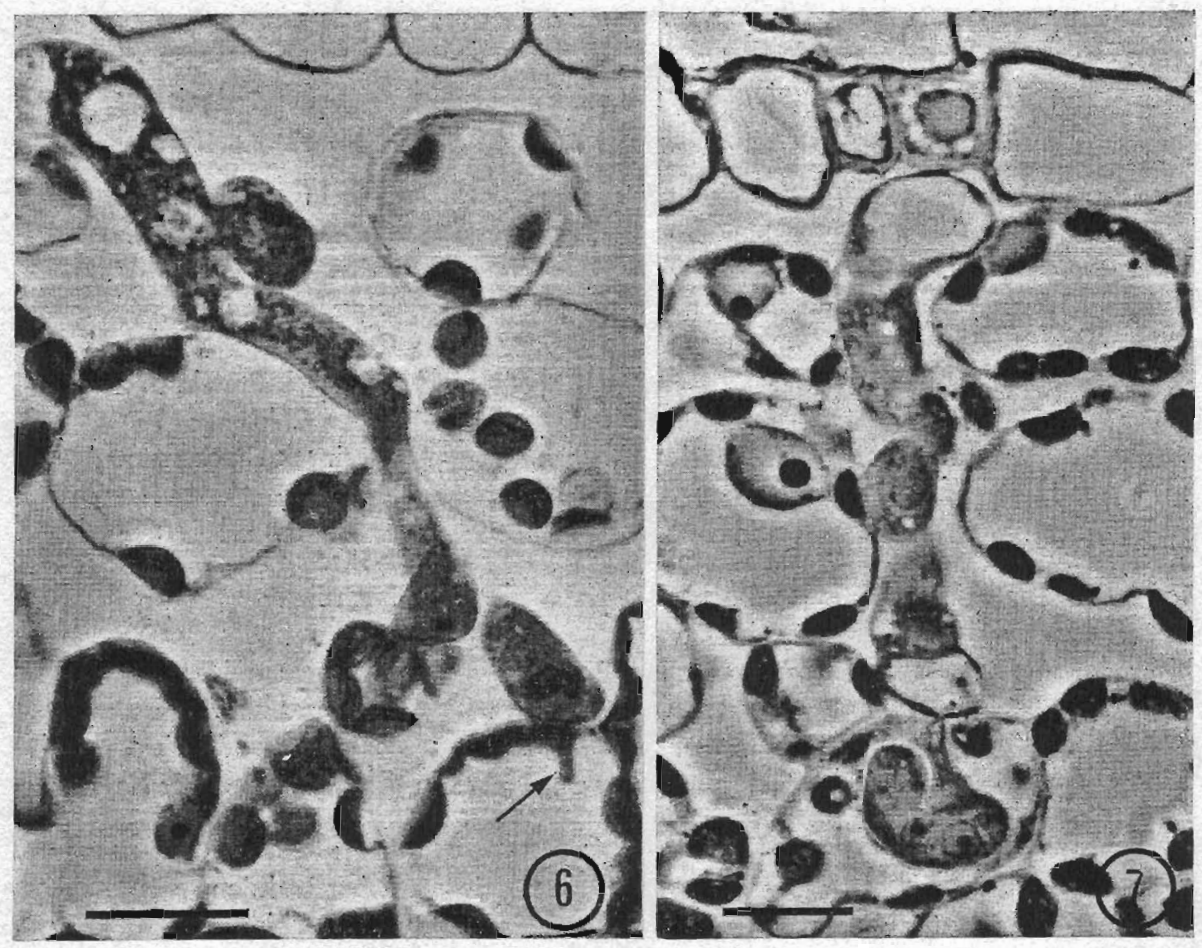

Abb.6. Die Infektionshyphe hat eine Haustorienmutterzelle ausdifferenziert, die mit dem Penetrationskeil (Pfeil) in die Wirtszelle eindringt (18 h.p.i.). - Abb.7. Infektionshyphe mit Haustorienmutterzelle und fertig ausdifferenziertem Haustorium, 21 h.p.i.

schließlich das ganze Appressorium erfüllt (Abb. 5). Anschließend sind der Infektionskeil und das substomatäre Vesikal durch ein Septum unterteilt (Abb. 5, MENDGEN 1973). Die Infektionshyphe, sie ist eine Verlängerung des substomatären Vesikels, wächst bis in die Mitte des Blattes (Grenzzone von Schwamm- und Palisadenparenchym) und verzweigt sich dort (Abb. 6). Der eine Arm der Infektionshyphe ist regelmäßig in Kontakt mit einer Wirtszelle und wird durch ein Septum von der Infektionshyphe abgeteilt. Dadurch entsteht eine Haustorienmutterzelle. Etwa 16 h.p.i. dringt diese mit einem Penetrationskeil (Abb. 6) in die Wirtszelle ein und bildet das erste Haustorium aus. Das erste, fertig ausgebildete Haustorium mit zwei Kernen und leerer Haustorienmutterzelle (Abb. 7) wird 21 h.p.i. angetroffen. Es ist auch gut zu erkennen (Abb. 8), daß die Infektionshyphe vielkernig ist, $d$. h. es müssen mehrere Kernteilungen stattgefunden haben.

Das zweite Haustorium, das wie das erste Haustorium entsteht, ist 28 h.p.i. fertig ausgebildet (Abb. 8), das dritte und vierte Haustorium etwa 12 Stunden später.

Die Entwicklung des Pilzes bei 21, 28 und 40 h.p.i. zeigt Abbildung 9 schematisch. Jede Zeichnung beruht auf mindestens zehn beobachteten Infektionsvorgängen. Dieses Schema soll als Vergleich für die nachfolgend beschriebenen Infektionsvorgänge bei den resistenten Bohnensorten dienen. 
3. Untersuchung des Infektionsverlaufs bei den resistenten Bohnensorten

Im Vergleich zu den Beobachtungen bei der anfälligen Bohnensorte Favorit stellten wir im Gewebe der Sorten Redlands Greenleaf A, Redlands Greenleaf B, Redlands Greenleaf C, NB 518, 07054, 010, 5961, 07036 und Epicur keinen Unterschied im Infektionsverlauf und in der Verträglichkeit der Wirtszellen im Untersuchungszeitraum (bis 28 h.p.i.) fest. Bei den anderen Bohnensorten beobachteten wir im Untersuchungszeitraum deutliche Unterschiede in der Reaktion der Wirtszellen auf die Infektion. Diese Unterschiede traten im Zeitraum von 21 bis 40 h.p.i. auf. Die Abbildungen stellen den Infektionsvorgang in diesem Zeitraum dar.

Abb. 8. Darstellung der Infektion, etwa 28 h.p.i. Es sind zwei Haustorien (Pfeile) im Schnitt getroffen, ein Teil der Hyphen liegt außerhalb der Schnittebene

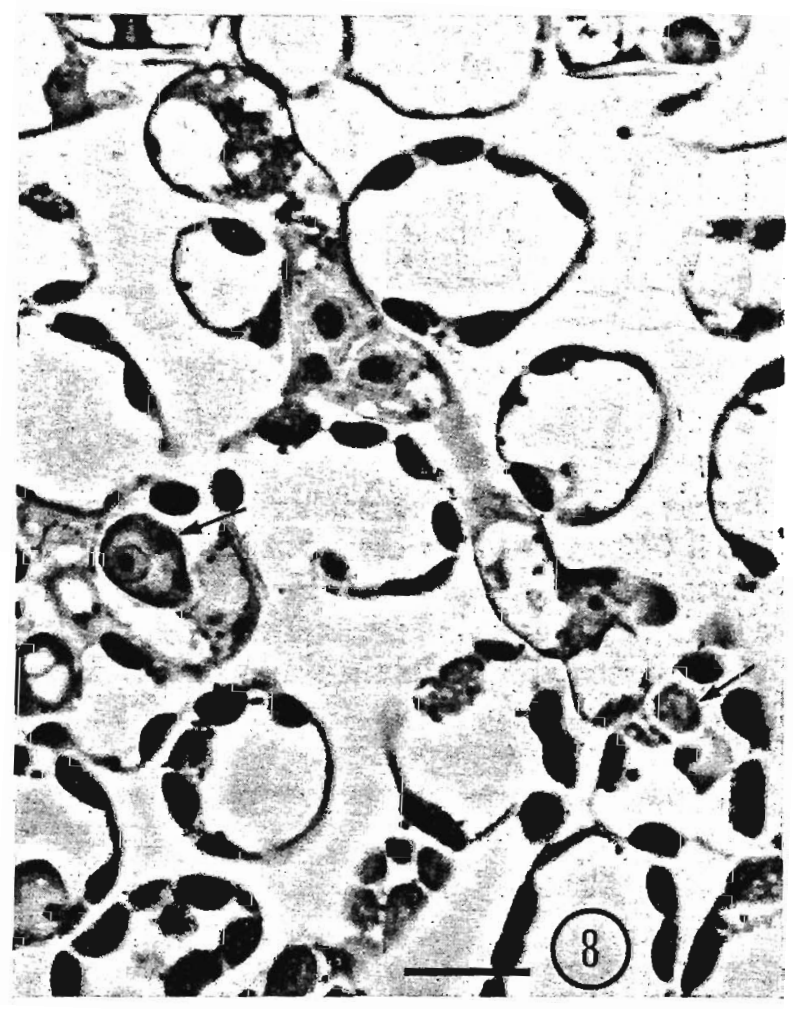

Die Zellen einiger Bohnensorten reagierten auf die Infektion durch den Rostpilz sehr ähnlich. Diese Sorten wurden zu Gruppen mit folgenden typischen Merkmalen zusammengefaßr:

1. Der Inhalt der infizierten Zelle löst sich bald nach Ausbildung des Haustoriums gleichmäßig auf.

2. Der Zellinhalt löst sich erst 28 h.p.i., also nach einer Verweildauer des Haustoriums von etwa 6 bis 8 Stunden in der Zelle auf.

3. Die infizierte Zelle kollabiert sofort nach Eindringen des Haustoriums.

4. Die infizierten Zellen kollabieren erst nach 10 bis 20 Stunden nach Ausbildung des Haustoriums in der infizierten Zelle.

5. Das Haustorium kollabiert in der infizierten Zelle, wird dunkel und schrumpft.

Diese typischen Merkmale, als Reaktionstyp bezeichnet, wurden bei folgenden Bohnensorten beobachtet: 

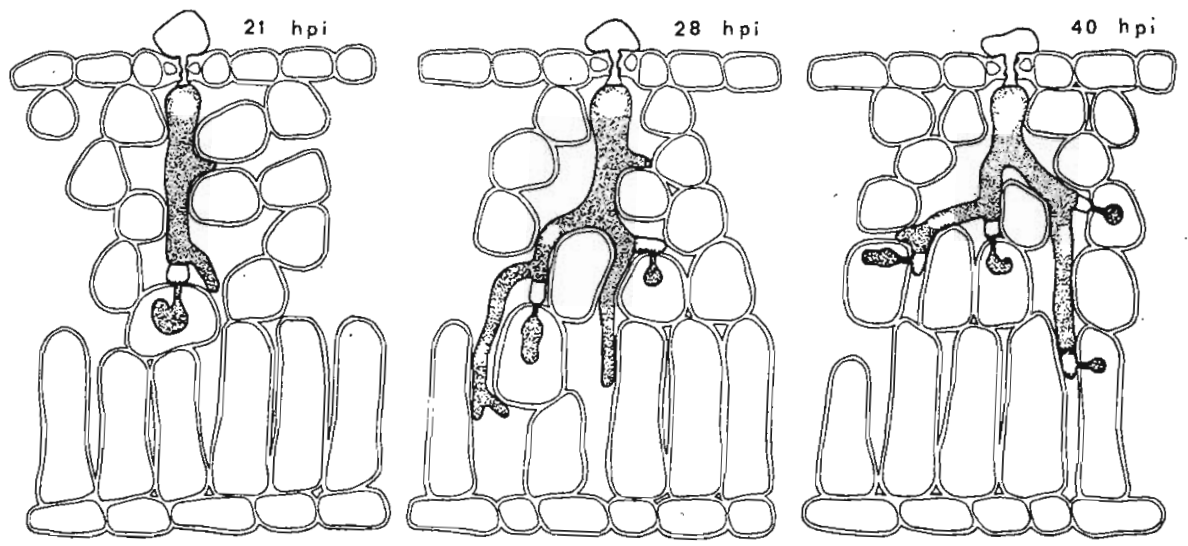

Abb. 9. Schematische Darstellung der Infektion bis 40 h.p.i.

\section{Reaktionstyp 1}

Die Infektionshyphe wächst bei den Bohnensorten Golden Gate Wax, 07054, 046 und 814 bis etwa in die Mitte des Blattes und differenziert eine Haustorienmutterzelle. Diese dringt mit dem Penetrationskeil in eine Wirtszelle ein und bildet ein Haustorium aus. Daraufhin schwellen die Plastiden in der infizierten und in einigen Nachbarzellen an und lösen sich ebenso wie der übrige Zellinhalt auf (Abb.10). Während 18 h.p.i. die meisten infizierten Zellen normal aussehen, sind etwa 21 h.p.i. von 40 infizierten Zellen 30 desintegriert. Trotzdem wachsen die interzellulären Hyphen des Pilzes langsam wei-
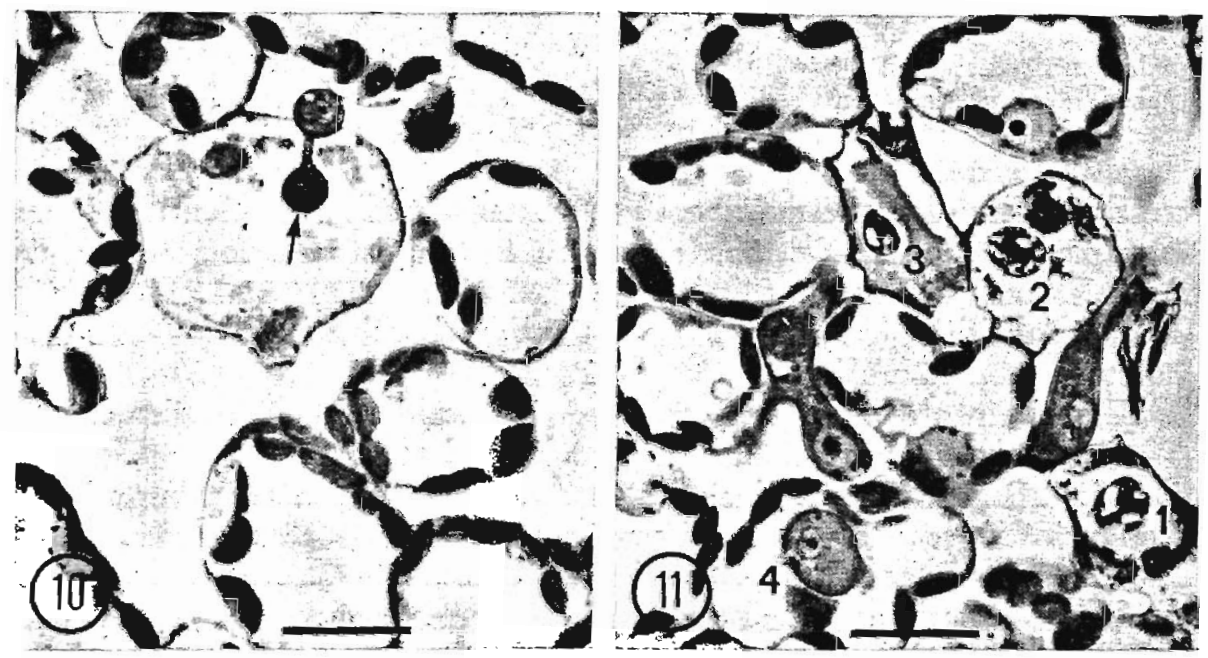

Abb. 10. Aufgelöster Inhalt der infizierten Zelle bei der Sorte Golden Gate Wax (Pfeil deutet auf Haustorium) 21 h.p.i. - Abb. 11. Haustorien in desintegrierten Wirtszellen (1-3) und normales Hustorium (4) in noch intakt aussehender Wirtszelle bei der Sorte 046 
ter. 28 h.p.i. sind die meisten infizierten Zellen und auch danebenliegende, nicht infizierte Zellen geschädigt. 40 h.p.i. wurden noch intakt aussehende Hyphen, aber nur noch wenige normal aussehende Zellen mit Haustorien angetroffen. Diese wurden in dem Schema der Infektion (Abb. 12) nicht mehr dargestellt. Offensichtlich können die Haustorien zunächst wenige Stunden in der Wirtszelle leben. Daraufhin löst sich der Plasmainhalt der Wirtszelle auf und anschließend stirbt auch das Haustorium. Währenddessen wächst die interzelluläre Hyphe weiter und differenziert andere Haustorienmutterzellen mit Haustorien aus. Diese sterben ebenfalls nacheinander wenige Stunden später. Das Ergebnis zeigt Abbildung 11. Hier befinden sich von den vier Haustorien, die von einer interzellulären Hyphe ausdifferenziert wurden, drei tot in den desintegrierten Zellen; Haustorium 4 erscheint dagegen normal und fertig ausdifferenziert in einer intakt aussehenden Wirtszelle.

\section{GOLDEN GATE waX}
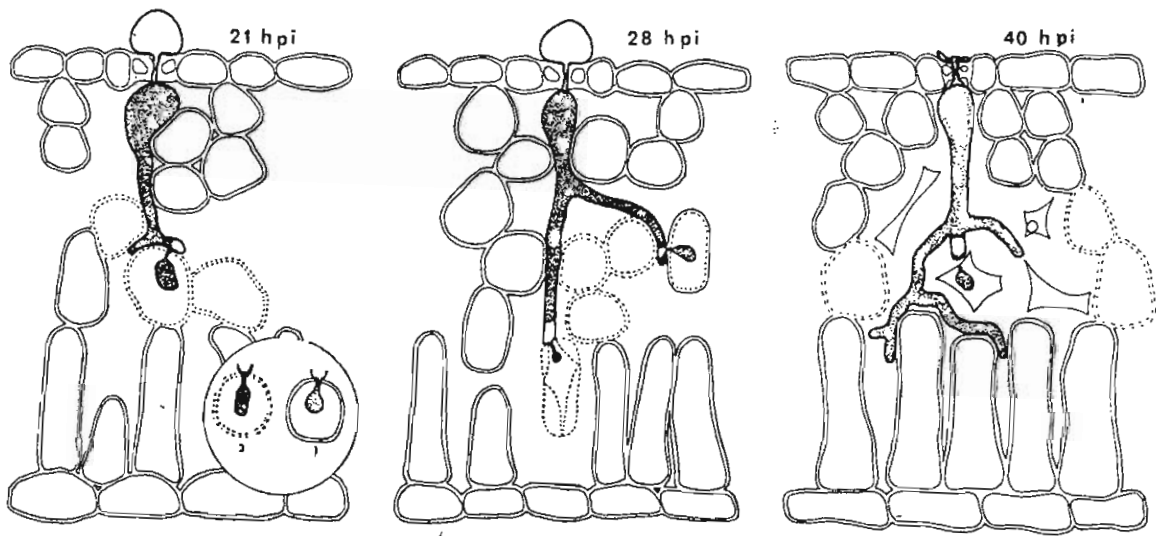

Abb. 12. Schematisch dargestellte Infektion bei der Sorte Golden Gate Wax.

Zellen mit aufgelöstem Inhalt sind gestrichelt gezeichner, kollabierte Zellen durchgezogen

\section{Reaktionstyp 2}

Bei der Bohnensorte Greenleaf löst sich der Zellinhalt wie beim Reaktionstyp 1 auf. Dieser Vorgang tritt jedoch erst 6 bis 8 Stunden später ein. Wirtszelle und Haustorium können also mindestens 6 Stunden ohne sichtbare Veränderungen miteinander existieren (Abb.13). Es fällt jedoch auf, daß sich in der Umgebung der infizierten Zellen der Inhalt einiger Parenchymzellen auflöst, selbst wenn wir diese nie in Kontakt mit der Pilzhyphe gesehen haben. Manchmal desintegrieren die Zellen im Infektionsbereich schon vor der Parenchymzelle, in die das Haustorium eingedrungen ist.

\section{Reaktionstyp 3}

Bei den Bohnensorten 08, 057 und Chiapas sterben die Wirtszellen ebenfalls schon kurz nach der Ausbildung des ersten Rosthaustoriums ab. Bei diesen 

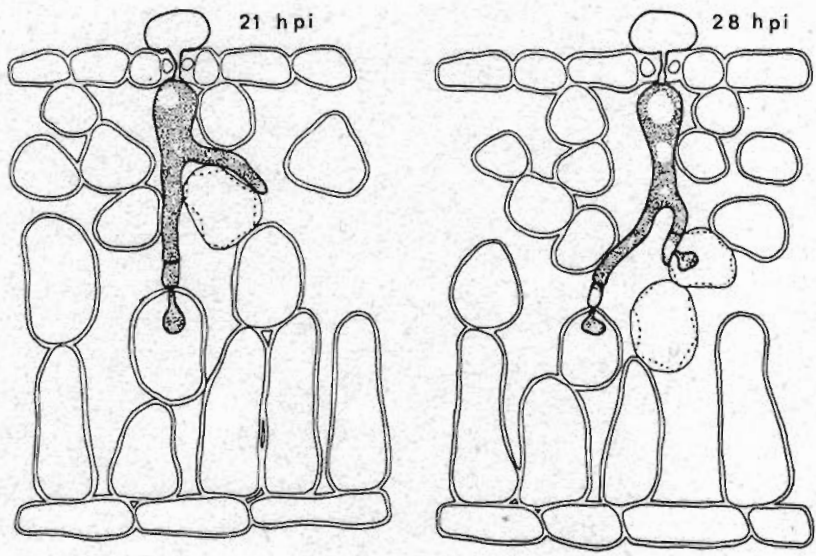

Abb. 13. Schema der Infektion bei der Sorte Greenleaf. Zellen mit beginnender Desintegration sind gestrichelt

Sorten beginnt die Zellnekrose jedoch durch Plasmolyse des Protoplasten (Abb. 14): Die Plastiden scheinen zu schrumpfen und die ganze Zelle, einschließlich des Haustoriums, kollabiert zu einer kompakten Masse (Abb. 15). Während die Infektionshyphe noch gut zu erkennen ist (Abb.15), sind die in-

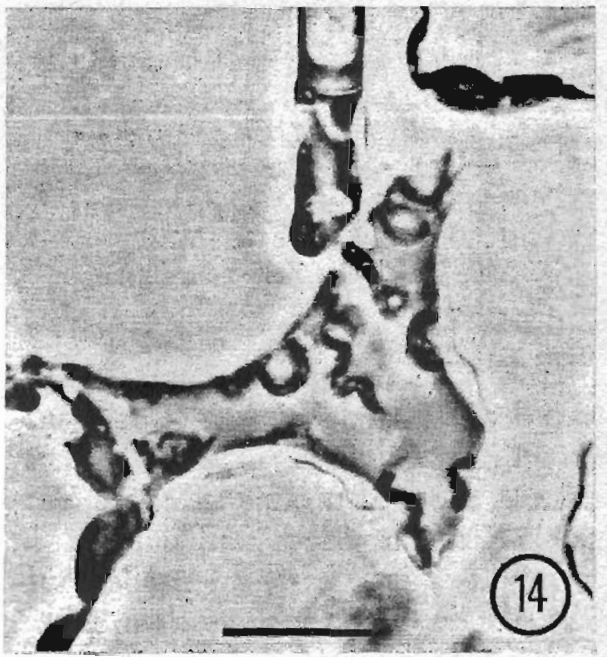

Abb. 14. Kollabierender Zellinhalt bei der Sorte 08 während der Ausbildung des Rosthaustoriums, 21 h.p.i.

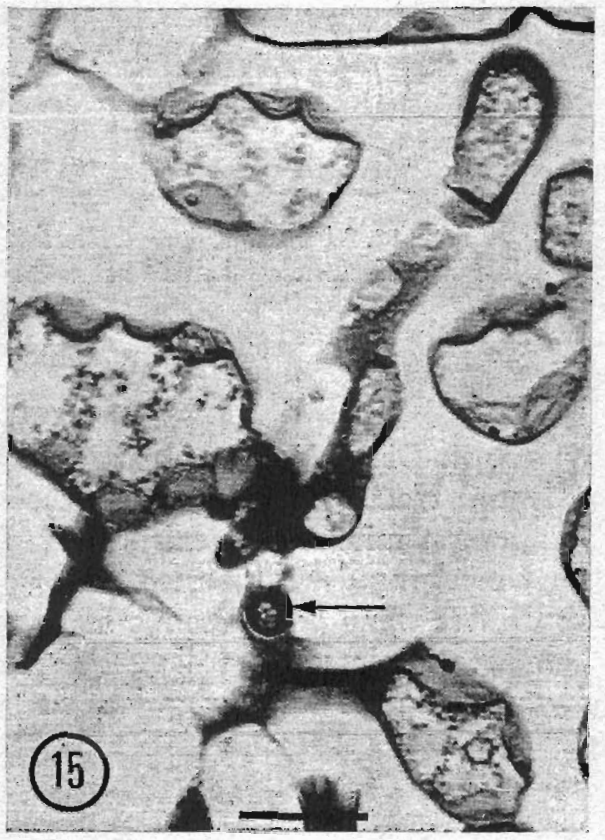

Abb. 15. Infektionsbereich im Blattparenchym bei der Sorte 057, 21 h.p.i. Der Pfeil deutet auf ein Haustorium in kollabierter Zelle 
fizierten Zellen (man erkennt sie am geschrumpften Haustorium) und meist noch einige Nachbarzellen kollabiert. Hier liegen außerdem noch sortenspezifische Unterschiede vor. Während bei den Sorten 08 und 057 (Abb.16) meist nur die infizierte Zelle kollabiert, sind es bei der Sorte Chiapas (Abb.17) im Infektionsbereich meist mehrere Zellen, deren Inhalt sich teils auflöst, teils kollabiert.

\section{Reaktionstyp 4}

Bei der Sorte 017 werden die Haustorien bis etwa 28 h.p.i. ohne sichtbare Schäden der Wirtszelle ausgebildet. Manchmal beobachter man eine Anhäufung von mitochondrienähnlichen Gebilden um die Haustorien. Erst zwischen
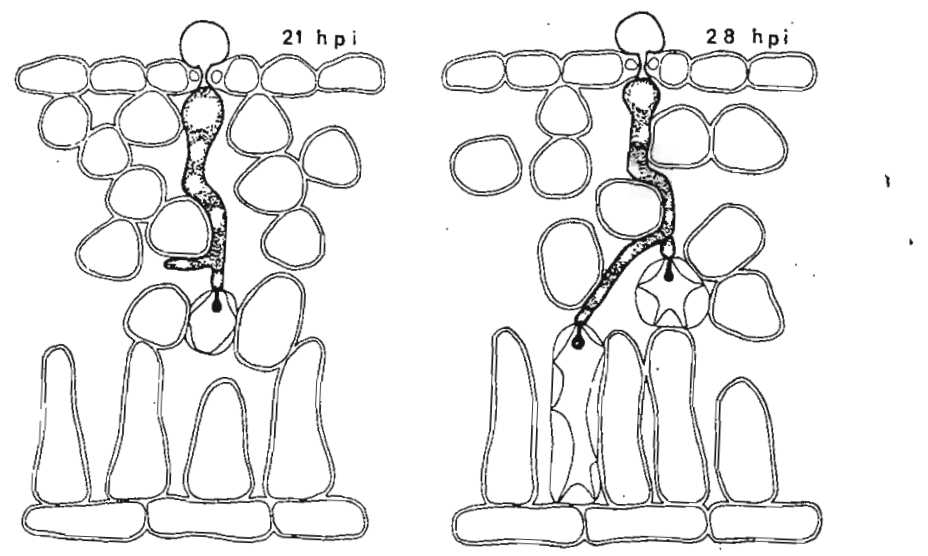

Abb. 16. Der Infektionsverlauf bei der Sorte 057.

Der kollabierte Zellinhalt ist durdıgezogen gezeichnet, tote Haustorien schwarz

CHIAPAS
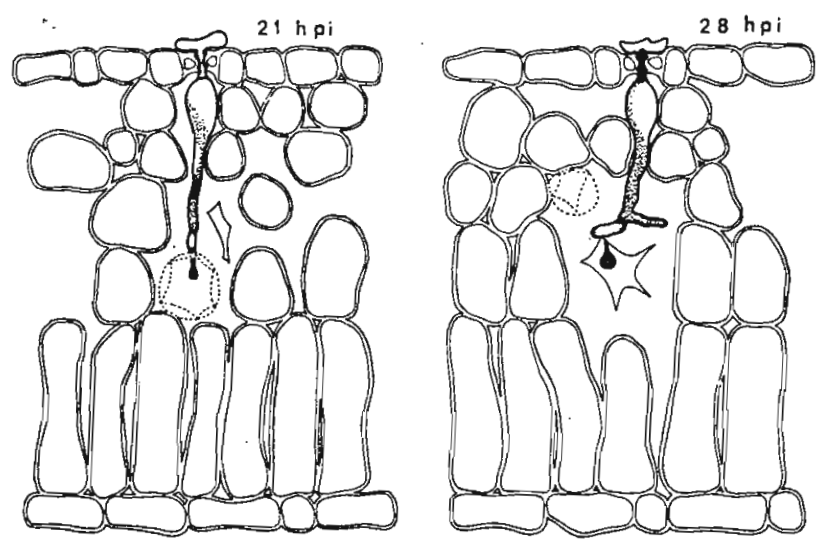

Abb.17. Der Infektionsverlauf bei der Sorte Chiapas.

Aufgelöste Zellen sind gestrichelt, kollabierte Zellen durdigezogen gezeichnet 
28 h.p.i. und 40 h.p.i. schwellen die Plastiden zunächst an, kollabieren aber dann ebenso wie die gesamte Zelle. Ahnlich reagieren die Nachbarzellen. 40 h.p.i. findet man nur noch Reste der Infektionshyphe (Abb. 18). Bei der Sorte Warox (Abb.19) tritt dieser Vorgang etwas später, etwa 40 h.p.i. und danach auf. Hier fällt wieder auf, daß nicht nur die infizierte, sondern ebenso die Zellen in der Umgebung auf ähnliche Art und Weise kollabieren. Die Zellnekrose ähnelt also der Zellnekrose bei Reaktionstyp 3, sie tritt nur sehr viel später auf und zu einem für jede Sorte typischen Zeitpunkt. Zur gleichen Zeit kollabieren nicht nur die Zellen und die Haustorien, sondern auch ein großer Teil der Hyphen.

\section{Reaktionstyp 5}

Bei den Bohnensorten Tui und Ciat beobachteten wir eine normale Haustorienausbildung bis 21 h.p.i. 28 h.p.i. jedoch sind die Haustorien kollabiert

017
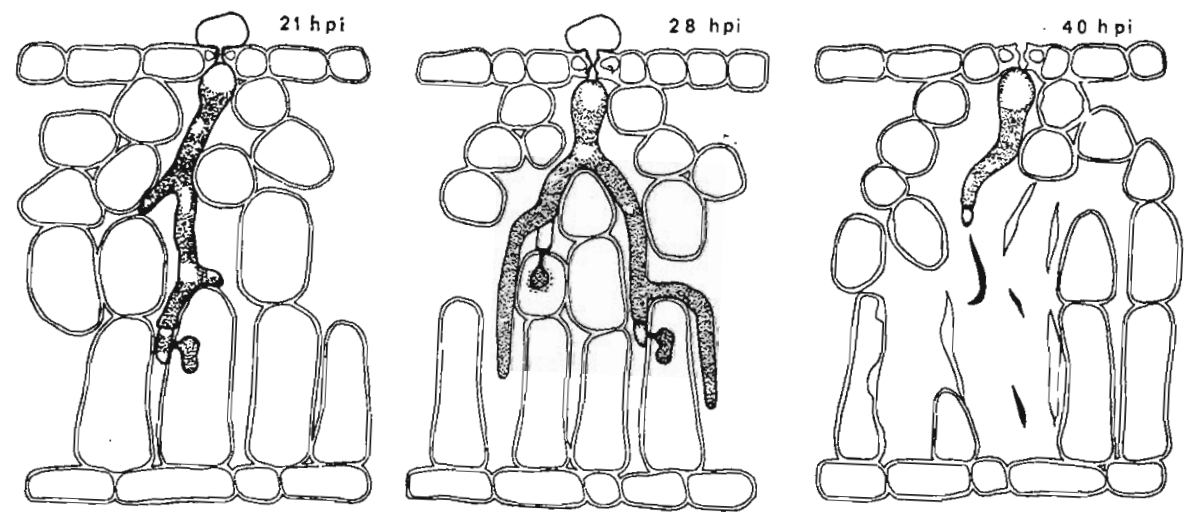

Abb. 18. Der Infektionsverlauf bei der Sorte 017

WAROX
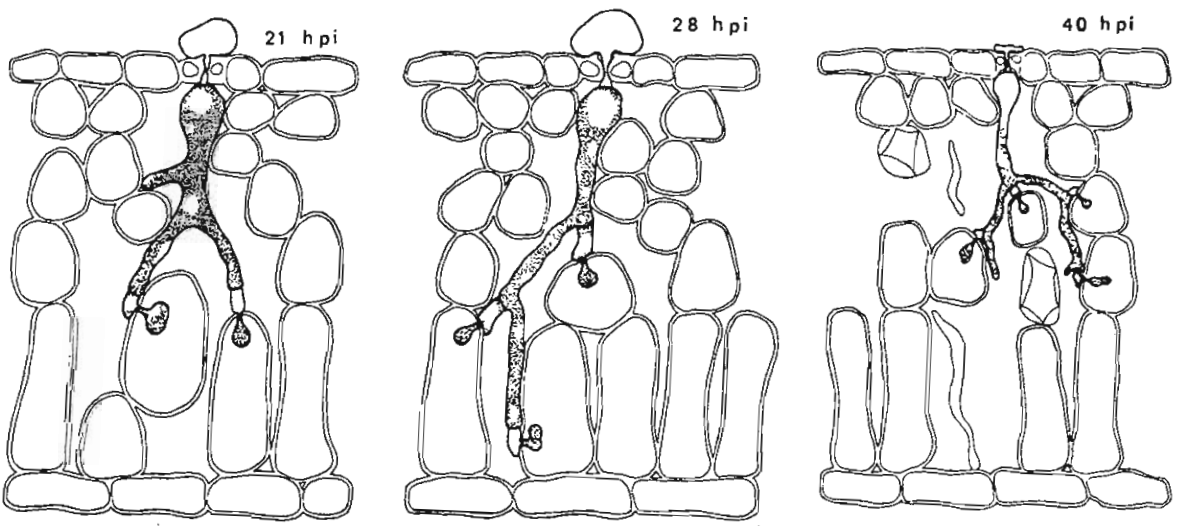

Abb. 19. Der Infektionsverlauf bei der Sorte Warox 
und gleichmäßig dunkel (Abb. 20). Auch die Haustorienmutterzelle schrumpft. Die Wirtszelle erschien im Untersuchungszeitraum (bis 28 h.p.i.) ohne krankhafte Veränderungen. Sie ist offensichtlich in der Lage, zunächst einmal das Haustorium abzukapseln ohne selbst zu degenerieren. Der weitere Verlauf der Interaktionen wurde nicht untersucht.

Abb. 20. Kollabiertes Haustorium bei der Sorte Tui (Pfeil)

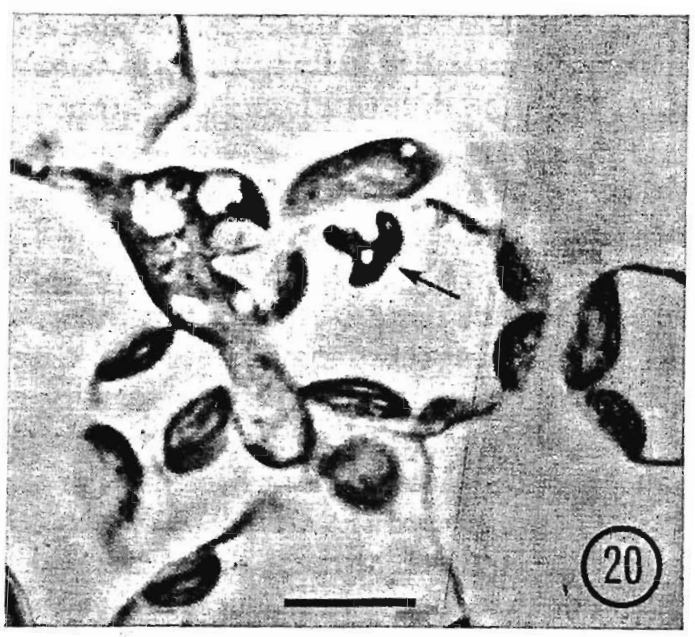

TUI
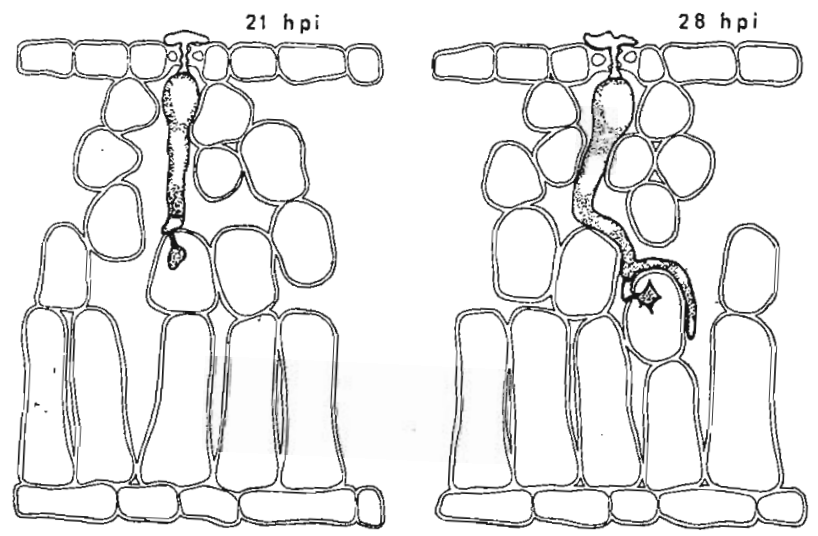

Abb. 21. Der Infektionsverlauf bei der Sorte Tui

\section{Diskussion}

Unsere Beobachtungen des Infektionsverlaufs von $U$. phaseoli erstreckten sich nicht auf die Keimung der Uredospore, das Wachstum des Keimschlauches zur Spaltöffnung hin und die Induktion des Appressoriums. Man kann jedoch annehmen, daß dieser Vorgang ähnlich wie bei dem verwandten Rostpilz Uromyces phaseoli var. vignae, auf Vigna sinensis (HEATH 1974), abläuft. Im weiteren Verlauf der Infektion zeigt der Bohnenrost jedoch einige Besonderheiten: 
Im Gegensatz zum Gelbrost (SCHRÖDER und Hassebrauk 1964), der kein Appressorium ausbildet, und im Vergleich zu anderen Rostpilzen (Evans 1907) differenziert der Bohnenrost ein besonders deutliches Appressorium aus. Darin findet, wie STAPLES et al. (1975) bei künstlich induzierten Appressorien gezeigt haben, die erste Kernteilung statt. In unseren Dünnschnitten waren die Kerne nur schlecht im dichten Plasma zu erkennen.

Wenn der Infektionskeil des Appressoriums die Schließzellen auseinanderdrückt (siehe MENDGEN 1973), entstehen im Appressorium kleine Vakuolen wie bei einigen Pilzen nach Ausbildung von Ruhestadien kurz vor der Infektion (siehe AIst 1976). Vielleicht wird dadurch ein Druck im Appressorium erzeugt, um das Plasma des Pilzes durch den Infektionskeil in das substomatäre Vesikel zu drücken. Das substomatäre Vesikel ist, anders als z. B. bei Puccinia graminis (Allen 1923, Evans 1907) etwa eiförmig und ragt mit seiner Längsachse in die Atemhöhle. Die mehrkernige Infektionshyphe, die sich meist erst in der Blattmitte gabelt, wächst nicht in Richtung der nächstliegenden Parenchymzellen wie bei anderen Rosten (Evans 1907, WARD 1903), sondern senkrecht zur Blattoberfläche in die Blattmitte und differenziert erst dort die Haustorienmutterzelle und das Haustorium aus. Dies geschieht im Zeitablauf in etwa wie bei den anderen Rostpilzen (LitTLEFIELD 1972, SkIPp und SAMBORSKI 1974).

Bei den untersuchten unverträglichen Wirtspflanzen erschien die Appressorienausbildung, die Differenzierung der Infektionshyphe und der Haustorienmutterzelle nicht gestört. Dies steht im deutlichen Gegensatz zu Beobachtungen von Rostinfektionen bei Nichtwirtspflanzen ( $z$. B. LEATH und RowelL 1969, HEATH 1974, 1977), wo schon zu diesem frühen Zeitpunkt der Infektion Veränderungen der Wirtszellen und der Infektionsstrukturen des Pilzes festgestellt wurden.

Bei den unverträglichen Wirtspflanzen kommt es erst bei und nach der Ausbildung der Haustorien zu Unverträglichkeitsreaktionen. Ahnliche Beobachtungen liegen für viele Rostpilze vor (HEATH 1974, LitTLEFIELD und Aronson 1969, Ogre und Brown 1971, Rothmann 1960, Skipp und SambORSKI 1974). Wie beim Rost des Safflors und beim Maisrost (HILU 1965, ZIMMER 1965) sterben auch bei der Bohne nach Infektion der unverträglichen Wirtszelle öfters Nachbarzellen im Infektionsbereich ab. Die Reaktionen der infizierten Zellen können, ähnlich. wie beim Flachsrost (LitTLEFIELd und Aronson 1969) und beim Haferkronenrost (RothmanN 1960) zu verschiedenen Zeitpunkten während des Infektionsverlaufs einsetzen.

Offensichtlich finden die Vorgänge im infizierten Gewebe, also die Unverträglichkeitsreaktionen der Wirtszellen, meist viele Stunden oder Tage vor der Ausprägung der Blattsymptome statt. So kann bei der Sorte Golden Gate Wax die Lyse des Inhalts der infizierten Zelle schon 21 h.p.i. einsetzen, eine makroskopisch sichtbare Chlorose des Blattgewebes aber erst am dritten Tag beobachtet werden. Möglicherweise stellen also die Blattsymptome wie Chlorose oder Nekrose (siehe Tab. 1) nur ein Sekundärsymptom der Pflanze dar.

Durch die praktisch artefaktfreie Einbettung unserer Proben konnten wir nun schon im Lichtmikroskop erkennen, daß die zu verschiedenen Zeitpunkten einsetzenden Reaktionen der Wirtszellen (Reaktionstypen) sehr unterschiedlich 
sind. Diese gut gegeneinander abgrenzbaren Möglichkeiten des Zelltodes deuten auf verschiedene Resistenzmechanismen oder Abwehrreaktionen der Pflanze, die ein weiteres Wachstum der Pilze behindern könnten.

Es ist naheliegend, daß hier die Wirkung der verschiedenen Resistenzgene der Pflanze und der Virulenzgene des Parasiten angezeigt wird. Das Eingreifen der Resistenzgene in die Wirt-Parasit-Interaktion haben PERSON und SrDHU (1974) als „Stop-Signal“ für die weitere Entwicklung der Wirt-Parasit-Beziehungen bezeichnet. HEATH (1974) schlägt vor, die Reaktion, bei der die WirtParasit-Kombination beginnt unverträglich zu reagieren, als "switching point ${ }^{\text {“ }}$ zu bezeichnen. Als Beispiele werden Zellwandveränderungen am Hals des Haustoriums oder Modifikationen des Plasmalemmas angeführt (HeATH 1974).

Wir möchten hier in Anlehnung an die Bezeichnung von HeatH (1974) den Ausdruck Kippunkt vorschlagen, bei dem, gesteuert durch die Resistenzund Virulenzgene, das Wirt-Parasit-Verhältnis auf spezifische Art und Weise und zu einem bestimmten Zeitpunkt auf unverträglich umkippt. Dieser Ausdruck soll auch deutlich machen, daß diese Unverträglichkeitsreaktionen eintreten können, aber nicht müssen. Im Rahmen einer großen Zahl vop Infektionsvorgängen sind auch andere Reaktionen möglich (siehe HEATH 1977).

Die Mechanismen an den Kippunkten, die das bis dahin verträgliche WirtParasit-Verhältnis beenden, müssen erst noch aufgeklärt werden. Erste Ergebnisse bei der Sorte Golden Gate Wax zeigen, daß, bevor sichtbare Schäden in der Wirtszelle zu beobachten sind, funktionelle Störungen des Haustoriums; die durch eine verringerte Substrataufnahme angezeigt werden, auftreten (MENDGEN 1977). Der Kippunkt, bei dem die Unverträglichkeit einsetzt, liegt also hier bei der Ausbildung der für die Substrataufnahme verantwortlichen Struktur.

Diese funktionelle Störung des Haustoriums tritt bei einer Bohnensorte auf (Golden Gate Wax), die dem Pilz ein weiteres Wachstum und eine stark verringerte Sporulation ermöglicht. Das Auflösen des Zellinhalts des Wirtes im weiteren Verlauf der Haustorienentwicklung ist offensichtlich von sekundärer Bedeutung. Möglicherweise auch die daraus resultierende geringere Pilzentwicklung im Gewebe, wie sie neuerdings auch beim Weizen mit "slow-rusting"Eigenschaften (MARTIN et al. 1977) beobachtet wurde. Hier sind weitere Untersuchungen notwendig um aufzuklären, ob die funktionelle Störung des Haustoriums (Hungern des Haustoriums) mit dem "slow rusting"-Phänomen (verringerte Sporenproduktion), das für die Züchtung neuer Sorten von besonderer Bedeutung ist, zusammenhängt.

Bei einigen resistenten Bohnensorten, wie z. B. bei der Sorte 017, die auf die Infektion mit Blattnekrosen reagiert und keinerlei Sporulation des Pilzes ermöglicht, scheint die Wirt-Parasit-Beziehung zu einem anderen Zeitpunkt auf unverträglich umzukippen. Die Haustorien sind zu einer normalen Stoffaufnahme in der Lage (MENDGEN, unveröffentlicht). Sie leben bei dieser Bohnensorte zunächst längere Zeit in der Zelle, ohne daß sichtbare Schäden eintreten. Es scheinen also erst verschiedene Stoffwechselvorgänge abzulaufen, bis der Tod von Pilz und Wirtszelle eintritt. Der Kippunkt, an dem diese Vorgänge eingeleitet werden, kann zunächst nur durch den Zeitpunkt, also bei der 
Sorte 017 etwa $z$ wischen 28 und 40 h.p.i., angegeben werden. Hier könnte eine histochemische Untersuchung der Enzymaktivitäten in der infizierten Zelle während der Ausdifferenzierung des Haustoriums weiterhelfen (MENDGEN 1975). Außerdem wäre zu überprüfen, ob das Haustorium den Zelltod induzieren kann (siehe MendGen und Heitefuss 1975).

Die Unverträglichkeit könnte auch an mehreren Kippunkten geregelt werden. Dies würde bedeuten, daß der Pilz am ersten Kippunkt nur in seiner Entwicklung gebremst wird und erst im weiteren Verlauf der Infektion durch weitere Zell reaktionen abgetötet wird. Diese Möglichkeit wurde auch schon beim Bohnenrost diskutiert: SEmpro et al. (1975) vermuten, daß die Reaktion der infizierten Zelle zwar das Haustorium, aber nicht den Pilz, d. h. die interzellulären Hyphen abtötet. Erst durch den weiteren Stoffwechsel, wie Anhäufung von Phenolkörpern, die hohe Aktivität von Peroxidasen und die veränderte Zusammensetzung der Aminosäuren führen schließlich zum Tod des Rostpilzes. Zu diesem späteren Zeitpunkt mögen dann auch Phytoalexine, die inzwischen ebenfalls bei der Sorte 017 nach Infektion durch den Bohnenrost nachgewiesen wurden (siehe EINAGHY und HerTEFuss 1976), für den weiteren Verlauf der Wirt-Parasit-Beziehungen von Bedeutung sein.

Weitere, ganz anders funktionierende Mechanismen, die zur Unverträglichkeit führen, scheinen bei den lateinamerikanischen Sorten Tui und Ciat vorzuliegen. Der Kippunkt, bei dem das Abtöten des Haustoriums in der zunächst noch intakten Zelle eingeleitet wird, scheint schon beim Beginn der Haustoriendifferenzierung zu liegen und ein Abkapseln des Haustoriums auszulösen. Als Folgereaktionen sterben dann die Zellen im Infektionsbereich ab und es entsteht die Nekrose. Eine ähnliche Reaktion der Wirtszelle wird manchmal auch bei anfälliger Kombination, besonders häufig aber nach Behandlung des Blattes mit Oxycarboxin beobachtet (PrING und Richmond 1976).

Die Wirkungsweise dieses Abwehrmechanismus, ebenso die Unverträglichkeitsreaktion der übrigen resistenten Sorten unseres Sortiments müssen noch genauer untersucht werden, um Klarheit darüber zu erhalten, wann und genau wie, also an welchen Kippunkten die entscheidenden Interaktionen zwischen Wirt und Parasit einsetzen, die zur Resistenz führen.

\section{Zusammenfassung}

Der Infektionsverlauf des Bohnenrosts und die Symptome des Primärblattes der Bohne nach Infektion werden bei der anfälligen Sorte Favorit und bei 20 resistenten Bohnensorten beschrieben.

Die Unverträglichkeitsreaktionen beginnen bei den resistenten Bohnensorten mit der Ausbildung der Haustorien in den Parenchymzellen der Wirtspflanze. Die Reaktionen waren bei einigen Bohnensorten sehr ähnlich. Bohnensorten mit ähnlichen Reaktionen wurden zu Gruppen mit folgenden typischen Merkmalen zusammengefaßt:

1. Reaktionstyp.

Der Inhalt der infizierten Zelle löst sich bald nach Ausbildung des Haustoriums auf. 
2. Reaktionstyp

Der Zellinhalt löst sich erst 28 h.p.i., also nach einer Verweildauer des Haustoriums von etwa 6 bis 8 Stunden in der Zelle auf.

3. Reaktionstyp

Die infizierte Zelle kollabiert sofort nach Eindringen des Haustoriums.

4. Reaktionstyp

Die infizierten Zellen kollabieren erst nach 10 bis 20 Stunden nach Ausbildung des Haustoriums in der infizierten Zelle.

5. Reaktionstyp

Das Haustorium kollabiert in der infizierten Zelle, wird dunkel und schrumpft.

Diese Ergebnisse legen den Schluß nahe, daß es sich hier um unterschiedliche Resistenzmechanismen handelt. Die Interaktionen zwischen Wirt und Parasit, an der die für die Unverträglichkeit verantwortliche Reaktion einsetzt, wird Kippunkt genannt. Die Bedeutung des Kippunktes für den weiteren Verlauf der Wirt-Parasit-Beziehungen wird diskutiert.

\section{Summary}

\section{The Bean Rust Infection Process in Resistant and Susceptible Cultivars of Bean}

The bean rust infection process and the symptoms of the primary leaf after rust infection are described in the susceptible cultivar Favorit and in twenty resistant cultivars.

In the resistant cultivars, the incompatibility reactions of the host cell begin with the formation of haustoria in the host parenchyma cells. In some bean cultivars, these reactions were very similar. Such cultivars were grouped together. The following groups with typical reactions were observed:

Reaction type 1

The content of the infected cell dissolves soon after formation of the haustorium in this cell (cv. Golden Gate Wax, 07054, 046, 814).

Reaction type 2

The content of the cell dissolves about 6-8 hours after formation of the haustorium in the host cell (cv. Greenleaf).

Reaction type 3

The infected cell and its content collapse at once after the penetration of the fungus (cv. 08, 057, Chiapas).

Reaction type 4

The infected cell and its content collapse about 10-20 hours after haustorial formation (cv. 017, Warox).

Reaction type 5

The haustorium collapses in the infected cell, becomes dark and shrivels before any host cell damage can be recognised (cv. Tui, Ciat).

These results suggest that the different groups may demonstrate the action of different mechanisms for resistance. The mode of action of these reactions 
is discussed. The interaction that is responsible for the incompatibility and induces it is called "Kippunkt".

Frau Prof. Dr. Michile Heath, Henrn Prof. Dr. W. H. Fuchs und Prof. Dr. R. Hertefuss danke ich für die Durchsicht des Manuskripts. Frau Ulrire Rose danke ich für die sorgfältige und selbständige technische Assistenz.

Der Deutschen Forschungsgemeinschaft danke ich für eine Sachbeihilfe.

\section{Literaturverzeichnis}

Arst, J. R., 1976: Cytology of penetration and infection-fungi. In: R. Heitefuss and P. H. Wrllams (Eds.), Physiolgical Plant Pathology, 197-221. Springer-Verlag, Heidelberg.

Allen, R. F., 1923: A cytological study of Baart, Kanred and Mindum wheats by Puccinia graminis forms III and XIX. J. Agric. Res. 26,131-152.

BURGA, C., O. VoYsest, and A. URBIYAGASTEgur, 1975: Dry bean international rust nursery 1974. Rep. Bean Improv. Coop. 18, 18-23.

Ellingboe, A. H., 1972: Genetics and physiology of primary infection by Erysiphe graminis. Phytopathology 62, 401-406.

Elnaghy, M. A., and R. Heitefuss, 1976: Permeability changes and production of antifungal compounds in Phaseolus vulgaris infected with Uromyces phaseoli. II. Role of phytoalexins. Physiol. Plant Path. 8, 269-277.

Evans, J. B., 1907: The cereal rusts. I. Development of their uredo mycelia. Ann. Bot, 21, $441-466$.

Heatr, M. C., and I. B. Heath, 1971: Ultrastructure of an immune and susceptible reaction of cowpea leaves to rust infection. Physiol. Plant Path. 1, 277-287.

- -, 1972: Ultrastructure of host and nonhost reactions to cowpea rust. Phytopathology 62, 27-38.

- -, 1974: Light and electron microscope studies of the interactions of host and non-host plants with cowpea rust - Uromyces phaseoli var, vignae. Physiol. Plant Path. 4, 403-414.

- -, 1977: A comparative study of non-host interactions with rust fungi. Physiol. Plant Path. 10,73-88.

Hilv, H. M., 1965 : Host pathogen relationships of Puccinia sorghi in nearly isogenic resistant and susceptible seedling corn. Phytopathology 55, 563-569.

LEATH, K. T., and J. B. ROWELL, 1969: Thickening of corn mesophyll cell walls in response

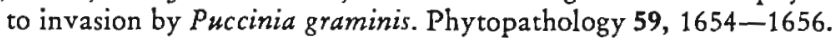

LitTlefield, L. J., and S. Y. Aronson, 1969: Histological studies of Melampsora lini resistance in flax. Canad. J. Bot. 47, 1713-1717.

- -, 1973: Histological evidence for diverse mechanisms of resistance to flax rust; Melampsora lini (Ehrenb.) Lev. Physiol. Plant Path. 3, 241-247.

Lupton, F. G. H., 1956: Resistance mechanisms of species of Triticum and Aegilops and of amphidiploids between them to Erysiphe graminis D.C. Trans. brit. mycol. Soc. 39, $51-59$.

Marte, M., e P. Montalbini, 1972: Microfluorescenza in foglie di fagiolo suscettibile e resistente alla ruggine. Phytopath. Z. 75, 59-73.

Martin, C. D., L. J. LitTlefield, and J. D. Miller, 1977: Development of Puccinia graminis f.sp. tritici in seedling plants of slow-rusting wheats. Trans. brit. mycol. Soc. 68, 161-166.

Merners, J. P., and C. W. Rogers, 1975: Reactions of bean cultivars and lines to three field collections of rust from Maryland. Rep. Bean Improv. Coop. 18, 47-48.

Mendgen, K., 1973: Feinbau der Infektionsstrukturen von Uromyces phaseoli. Phytopath. Z. $78,109-120$.

-, 1975 : Ultrastructural demonstration of different peroxidase activities during the bean rust infection process. Physiol. Plant Path. 6, 275-282. 
- - 1977: Reduced lysin uptake by bean rust haustoria in a resistant reaction. Naturwissenschaften $64,438-439$.

- - and R. HeItefuss, 1975: Microautoradiographic studies on host parasite interactions. I. The infection of Phaseolus vulgaris with tritium labeled uredospores of Uromyces phaseoli. Arch. Microbiol. 105. 193-199.

Murunya, D. M., 1975: Sources of resistance to bean anthracnose and bean rust in Kenya local dry beans. Rep. Bean Improv. Coop. 18, 49-51.

OGLE, H. Y., and J. F. BRoWN, 1971: Quantitative studies of the post-penetration phase of infection by $P$ uccinia graminis tritici. Ann. appl. Biol. 67, 309-319.

- - and C. Y. Johnson, 1974: Physiologic specialization and control of bean rust in Queensland. Queensland J. Agric. 31, 71-82.

Person, C., and G. M. E. Mayo, 1974: Genetic limitations on models of specific interactions between a host and its parasite. Canad. J. Bot. 52, 1339-1347.

Pring, R. J., and D. V. Richmond, 1976: An ultrastructural study on the effect of oxycarbonin on Uromyces phaseoli infecting leaves of Phaseolus vulgaris. Physiol. Plant Path. 8, 155-162.

RothManN, P. G., 1960: Host-parasite interactions of eight varieties of oats infected with race 202 or Puccinia coronata var. avenae. Phytopathology 50, 914_-918.

Schröder, G., und K. Hassebrauk, 1964: Untersuchungen über die Keimung der Uredosporen des Gelbrostes (Puccinia graminis West.). Zbl. Bakt., Abt. II, 622-657.

Sempto, C., G. Della Torre, F. Ferranti, B. Barberin1, and R. Draoli, 1975: Defence medhanism in beans resistant to rust. Phy topath. Z. 83, 244-266.

SKIPP, R. A., and D. Y. SAMBORSKr, 1974: The effect of the Sr6 gene for host resistance on histological events during the development of stem rưst in near-isogenic wheat lines. Canad. J. Bot. 52,1107-1115.

Staples, R. C., A. A. App, and P. Riccr, 1975: DNA synthesis and nuclear division during formation of infection structures by bean rust uredospore germlings. Arch. Mikrobiol. $104,123-127$.

Straib, W., 1943: Untersuchungen zur Biologie und Bekämpfung des Bohnenrostes (Uromyces phaseoli (Pers.) Wint. Gartenbauwissenschaft 17, 397-445.

WARD, M. H., 1903: On the histology of Uredo dispersa, Erikss., and the "mycoplasm" hypothesis. Phil. Transact. Roy. Soc. London S. B. 196, 29-46.

Zaumeyer, W. J., and G. P. Meiners, 1975: Disease resistance in beans. Ann. Rev. Phyropath. 13,313-334.

ZrMMER, D. E., 1965: Rust infection and histological response of susceptible and resistant Safflower. Phytoparhology 55, 296-301.

Anschrift des Verfassers: Priv.-Doz. Dr. Kurt Mendgen; Institut für Pflanzenpathologie und Pflanzeñschutz der Georg-August-Universität, Grisebachstraße 6, D-3400 Götringen. 